\title{
Androgen receptors expressed by prostatic stromal cells obtained from younger versus older males exhibit opposite roles in prostate cancer progression
}

\author{
You-Yi Lu ${ }^{1, *}$, Bo Jiang ${ }^{1,2, *}$, Fu-Jun Zhao ${ }^{1}$, Di Cui ${ }^{1}$, Qi Jiang ${ }^{1}$, Jun-Jie Yu ${ }^{1}$, En-Hui Li ${ }^{1}$, Xiao-Hai Wang ${ }^{1}$, \\ Bang-Min $\operatorname{Han}^{1}$ and Shu-Jie Xia ${ }^{1}$
}

Aging is a major risk factor for prostate cancer (PCa), and prostatic stromal cells may also promote PCa progression. Accordingly, stromal cells do not equally promote PCa in older males and younger males. Therefore, it is also possible that the expression of androgen receptors (ARs) by prostatic stromal cells in older versus younger males plays different roles in PCa progression. Using a gene knockdown technique and coculture system, we found that the knockdown of the AR in prostatic stromal cells obtained from younger males could promote the invasiveness and metastasis of cocultured PC3/LNCaP cells in vitro. By contrast, the invasiveness and metastasis of LNCaP cells was inhibited when cocultured with prostatic stromal cells from older males that when AR expression was knocked down. Moreover, after targeting AR expression with small hairpin RNA (shRNA), matrix metalloproteinase (MMP) expression in stromal cells was observed to increase in the younger group, but decreased or remained unchanged in the older group. One exception, however, was observed with MMP9. In vivo, after knocking down AR expression in prostatic stromal cells, the incidence of metastatic lymph nodes was observed to increase in the younger age group, but decreased in the older age group. Together, these data suggest that the AR in prostatic stromal cells played opposite roles in PCa metastasis for older versus younger males. Therefore, collectively, the function of the AR in prostatic stromal cells appears to change with age, and this may account for the increased incidence of PCa in older males.

Asian Journal of Andrology (2013) 15, 672-678; doi:10.1038/aja.2013.45; published online 24 June 2013

Keywords: age factors; androgen receptor; human; metastasis; prostate cancer

\section{INTRODUCTION}

Prostate cancer $(\mathrm{PCa})$ is the second most frequently diagnosed malignancy worldwide. ${ }^{1}$ In the United States, PCa is the most common malignancy diagnosed and the second leading cause of cancer-related death. ${ }^{2}$ The incidence of PCa increases with age, with the probability of developing PCa is $0.01 \%$ before the age of 39 years, $2.44 \%$ between the ages of 40 and 59 years, $6.45 \%$ between the ages of 60 and 69 years and $12.48 \%$ over the age of 70 years. ${ }^{2}$ In particular, the stromal microenvironment has been found to play an important role in the development of prostatic malignancy. ${ }^{3,4}$ For example, Xia et al. ${ }^{5}$ demonstrated that stromal cells from younger males and older males could both promote PCa progression. However, stromal cells from older males were more active.

Many articles have focused on the significance of androgen receptor (AR) expressed in epithelial cells. In the malignant process, the AR plays a very important role and serves as the basis for endocrine therapy. ${ }^{6}$ Accordingly, the AR expressed by prostatic epithelial cells have been hypothesized to promote PCa progression. ${ }^{7}$ Moreover, for cases involving the development of resistance, AR activation due to overexpression has been implicated as a potential mechanism. ${ }^{8}$ Previous studies have shown that inhibition of AR expression is in itself sufficient to induce cell death in both androgen-dependent and castration-resistant prostate cancer cells. ${ }^{9,10}$ However, in recent years, the role of the AR in stromal cells has received increasing attention. ${ }^{6,11}$ For PCa, AR progression is androgen dependent, and therefore, involves prostatic stromal AR and not epithelial AR. ${ }^{12}$ Correspondingly, Henshall et al. ${ }^{13}$ and Ricciardelli et al. ${ }^{14}$ demonstrated that loss of AR expression in the tumour stroma, but not in the surrounding normal prostatic stroma tissue, resulted in an increased risk of biochemical relapse following radical prostatectomy. ${ }^{13,14}$ Therefore, the loss of the stromal AR may influence both the aggressiveness of PCa and its response to castration therapy. ${ }^{15}$

PCa mostly affects older men worldwide. Therefore, the effects of age on the prostatic microenvironment may be an important factor in PCa progression. ${ }^{16}$ In our previous studies, peripheral zone (PZ) stromal cells from older males were found to enhance the proliferation, migration, and invasion of cocultured benign BPH-1 and PC-3 cells. In addition, older $\mathrm{PZ}$ stromal cells were more effective than younger $\mathrm{PZ}$ cells in promoting tumour formation in higher passage $\mathrm{BPH}-1$ cells (e.g., >100 passages) and PC-3 cells. ${ }^{5}$ To explain the differences between prostatic stromal cells in older males versus younger males, 
the role of AR expression by prostatic stromal cells in these two groups was investigated.

\section{MATERIALS AND METHODS}

Primary cultures of prostatic stromal cells and cell lines

The young donors were normal organ donors who died from traffic accidents, and the old donors were those who had bladder cancer and endured the operation with a resected prostate. Fresh prostate tissue was obtained, as previously reported, ${ }^{17}$ from the normal $\mathrm{PZ}$ of five younger male donors (aged 23, 25, 26, 30 and 32 years) and from five older male donors (aged 56, 61, 64, 71 and 75 years). All the donors had normal serum levels of total prostate specific antigen $(\leqslant 4 \mathrm{ng}$ $\left.\mathrm{ml}^{-1}\right)$. To further ensure the 'normal' status of prostate specimens, adjacent tissue around each specimen was stained with haematoxylin and eosin $(\mathrm{H} \& \mathrm{E})$, according to the procedure reported by Barclay et al., ${ }^{3}$ and specimens were examined by two pathologists. Collected tissue specimens were minced into pieces less than $1 \mathrm{~mm}^{3}$ in size and incubated with type I collagenase $\left(200 \mathrm{U} \mathrm{ml}^{-1}\right)$ for $8-10 \mathrm{~h}$. Specimens were then washed on a $149-\mu \mathrm{m}$ filter, transferred to $25-\mathrm{cm}^{2}$ flasks in $5 \mathrm{ml}$ of RPMI 1640 medium supplemented with $10 \%$ foetal bovine serum and antibiotics $\left(100 \mu \mathrm{g} \mathrm{ml}^{-1}\right.$ streptomycin and $100 \mathrm{IU} \mathrm{ml}^{-1}$ penicillin), and maintained at $37^{\circ} \mathrm{C}$ in $5 \% \mathrm{CO}_{2}$. All supplements were purchased from Gibco Invitrogen (Grand Island, NY, USA). All experimental protocols were approved by the Medical Ethics Committee of the Shanghai First People's Hospital (Shanghai, China) and have therefore been performed in accordance with the ethical standards established in the 1964 Declaration of Helsinki and its later amendments. LNCaP cells were purchased from the cell bank of the Chinese Academy of Sciences (Shanghai, China). PC3 cells were a kind gift from Professor Ju Zhang (Institute of Molecular Biology, College of Life Sciences, Nankai University, Tianjin, China).

\section{Preparation of lentivirus vector encoding small hairpin RNA (shRNA) and cell transfection}

Prostate stromal cells were passaged three times before being transfected with a lentivirus carrying an shRNA designed to target $A R$ mRNA. The use of an AR-targeting shRNA to effectively reduce levels of AR protein was described previously. ${ }^{14}$ A pGLV1/U6/GFP lentiviral vector expressing shRNA specific for human AR (GCACTGCTACTCTTCAGCA), as well as a non-target shRNA (CTCCGAAC GTGTCACGT), were obtained from GenePharma RNAi Company (Shanghai, China). Lentivirus production was performed by cotransfecting the pGLV1/U6/GFP vector and packaging plasmids (Shanghai GenePharma RNAi Company) into HEK293T cells using Lipofectamine 2000 (Invitrogen), according to the manufacturer's instructions. Supernatants containing lentiviral particles were harvested $48 \mathrm{~h}$ post-transfection, and the virus titre was determined using peripheral stromal cells. A multiplicity of infection of approximately 50 was used to achieve shRNA expression, and infections were performed with viral supernatant containing $5 \mu \mathrm{g} \mathrm{ml}^{-1}$ polybrene (Sigma). The transfection efficiency was found to be $>95 \%$, as observed under a fluorescence microscope. The levels of the $A R$ were also verified by quantitative PCR.

\section{Quantitative real-time polymerase chain reaction (q-PCR)}

Expression of matrix metalloproteinases (MMPs) was confirmed by qPCR using an ABI 7900 instrument. In addition, SYBR Green PCR Master Mix (Takara Biotech, Dalian, China) was used according to the manufacturer's recommendations. Primers for target genes were designed by the Sangon Company (China), and primer specificity was checked using Primer Blast in PubMed. Primer sets were designed as follows: AR, 5'-TGTACACGTGGTCAAGTGGGCCA-3' (sense) and 5'-GGGGCGAAGTAGAGCATCCTGGA-3' (antisense); matrix metalloproteinase 1 (MMP1), 5'-GAAGAATGATGGGAGGCAAGT-3' (sense) and 5' -GAGGACAAACTGAGCCACATC-3' (antisense); matrix metalloproteinase 2 (MMP2), 5'-AGAGACAGTGGATGATGCCTTT-3' (sense) and 5'-ATCGTCATCAAAATGGGAGTCT-3' (antisense); matrix metalloproteinase 7 (MMP7), 5'-GAGGCATGAGTGAGCTACAGTG-3' (sense) and 5'-CATCTCCTTGAGTTTGGCTTCT-3' (antisense); matrix metalloproteinase 9 (MMP9), 5'-ACCACCACAACATCACCTATTG-3' (sense) and 5' -ACACCAAACTGGATGACGATG-3' (antisense). Sequence detection system software (version 1.6.3; Applied Biosystems, Warrington, UK) was used for real-time data collection and analysis. Each target gene was normalized to levels of glyceraldehyde -3-phosphate dehydrogenase mRNA expression using the $2^{-\Delta \Delta C T}$ method. ${ }^{18}$

\section{Western blot analysis}

Stromal cells were transfected for $48 \mathrm{~h}$ in six-well plates and then were harvested and resuspended in $100 \mu \mathrm{l}$ of RIPA lysis buffer. Extracts were analysed by Western blotting, and the following antibodies were used: anti-human AR (1:1000; Cell Signaling Technology, Boston, MA, USA), anti-GAPDH ( $1: 1000$; Cell Signaling Technology) and antiMMP2 (1:1000; Santa Cruz Biotechnology, Dallas, TX, USA). Additionally, polyclonal antibodies were produced by immunising rabbits with a synthetic peptide (KLK-coupled) corresponding to residues surrounding Ser213 of the human androgen receptor. The blots were subsequently incubated with anti-rabbit or anti-mouse IgG horseradish peroxidase-conjugated antibodies $(1: 2000$; Cell Signaling Technology). Antibody binding was detected using standard enhanced chemiluminescence reagents and methods.

\section{In vitro coculture}

Using a two-chamber coculture system (Costar; Corning Inc., New York, NY, USA), stromal cells were maintained separate from epithelial cells (e.g., PC3 and LNCaP cells), and microporous filters $(0.4-$ or $8-\mu \mathrm{m}$ pore size) permitted the exchange of soluble factors between the two distinct chambers. All the experiments were performed in triplicate.

\section{Cell migration and invasion assays}

LNCaP and PC- 3 cells $\left(6 \times 10^{4}\right.$ cells per well) were seeded onto microporous $(8.0 \mu \mathrm{m})$ permeable inserts, whereas stromal cells $\left(2 \times 10^{5}\right.$ cells per well) were seeded in the lower chambers as chemoattractants. Cell invasion assays were conducted as described above except that the cell density in the upper chamber was $1.2 \times 10^{5}$ cells per well, and the microporous inserts were coated with $50 \mu \mathrm{l}$ of Growth Factor Reduced Matrigel (Becton Dickinson Biosciences, Bedford, MA, USA) diluted 1:3 with RPMI 1640 medium. After $12 \mathrm{~h}$, cells in the upper chamber were removed with a cotton swab, and cell culture inserts were fixed with $4 \%$ paraformaldehyde and stained with crystal violet. Cells that migrated to the bottom of each filter were counted for six representative microscopic fields at $\times 200$ magnification.

\section{Assessment of cancer metastasis in a nude mouse model}

Male nude mice, 6 weeks old, were purchased from the Animal Centre of the Chinese Academy of Sciences (Shanghai, China) and raised in a specific pathogen-free barrier facility according to our institutional guidelines. Stromal cells from different ages with or without AR and PC3 cells $\left(5 \times 10^{6}\right)$ were mixed in a $1: 1$ ratio and suspended in $50 \mu \mathrm{l}$ of undiluted Matrigel (Becton Dickinson Biosciences, Franklin Lakes, NJ, USA). Cell mixtures were then transplanted into the left prostate 
anterior lobe of male athymic mice. Seventy mice were separated randomly into five groups (PC3, PZ-young + PC3, AR siRNA PZyoung + PC3, PZ-old + PC3, AR siRNA PZ-old + PC3), and each group contained 14 mice. At the experimental end point, mice were sacrificed with $\mathrm{CO}_{2}$, and tumours were harvested. Tumescent lymph nodes were also measured, fixed with $10 \%$ formalin, and subjected to routine H\&E staining. Expression of MMP2 was detected by immunohistochemistry using an S-P technique, ${ }^{5}$ and counterstaining with diaminobenzidine was performed. Positive signals were quantified using Image Pro Plus 5.0 software. All experimental protocols were approved by the Medical Ethics Committee of the Shanghai First People's Hospital (Shanghai, China).

\section{Statistical analysis}

Values are expressed as the mean \pm s.d. All experimental procedures were performed in triplicate, and SPSS for Windows (Version 14.0; Chicago, IL, USA) was used to conduct statistical analyses. The $t$-test was used to compare values between two groups regarding in vitro experimental data, and the Kruskal-Wallis test was used to compare values regarding in vivo experimental data. Calculated $P$ values were two-sided, and a $P<0.05$ was considered statistically significant.

\section{RESULTS}

Stromal AR expression was inhibited by lentiviral-based shRNA Stromal cells were isolated from prostate tissue obtained from the normal PZ of five younger male donors and five older male donors. These cells were each passaged three times, and then were transfected with a lentivirus construct expressing an shRNA designed to target $A R$ mRNA. The efficiency of AR knockdown was measured by q-PCR and Western blot analysis. For stromal cells obtained from both the older and younger groups that were assayed $96 \mathrm{~h}$ after transfection by qPCR, levels of $A R$ mRNA were found to be inhibited by up to $94 \%$ (Figure 1a). Similarly, levels of stromal AR protein were also lower following transfection as measured by Western blotting (Figure 1b). In addition, the expression of AR in stromal cells did not significantly differ between the older and younger age groups.

Knockdown of AR expression in stromal cells from younger males promotes the migration and invasion of $\mathrm{PC} 3$ and LNCaP cells PC3/LNCaP cells and stromal cells were cultured in a two-chamber coculture system, and after $12 \mathrm{~h}$, the number of PC3/LNCaP cells that migrated through the membrane was counted. These assays demonstrated that all stromal cells with or without AR expression from the younger and older groups could promote the migration and invasion of PC3/LNCaP cells (Figure $\mathbf{2 b}$ and $\mathbf{c}$ and Figure $\mathbf{3 b}$ and $\mathbf{c}$ ).

Regarding the migration and invasion assays, PC3 cells cocultured with older PZ stromal cells were observed to transferred through the membrane at a faster rate than PC3 cells cocultured with younger PZ stromal cells (Figure $\mathbf{2 b}$ and $\mathbf{c}$ ). This finding was also demonstrated in our previous study. ${ }^{19}$ Moreover, there was no difference in the migration and invasion of PC3 cells that were cocultured with younger versus older PZ cells expressing AR-targeting shRNA (Figure 2b and c). However, PC3 cells cocultured with younger PZ stromal cells expressing AR-targeting shRNA did migrate and invaded at a faster rate than PC3 cells cocultured with younger PZ stromal cells (Figure $\mathbf{2 b}$ and $\mathbf{c}$ ). In addition, there was no significant difference observed between older PZ cells with and without expression of ARtargeting shRNA (Figure $\mathbf{2} \mathbf{b}$ and $\mathbf{c}$ ). For LNCaP cells, migration and invasion assays demonstrated that LNCaP cells cocultured with older PZ stromal cells transferred through the membrane more rapidly than

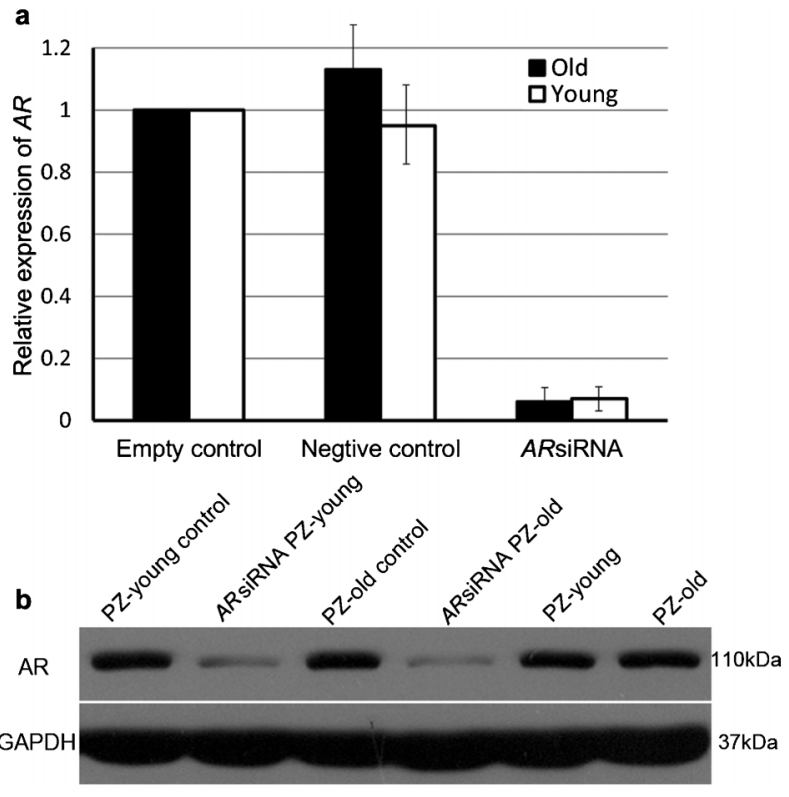

Figure 1 The AR was effectively inhibited by shRNA in prostate stromal cells from older and younger males. (a) Q-PCR analysis revealed that the knockdown efficiency for both groups was greater than $90 \%$. The value represents the mean \pm s.d. of three independent experiments. (b) Western blot analysis confirmed that expression of the AR in prostatic stromal cells was mostly inhibited following knockdown of the AR by shRNA. AR, androgen receptor; Q-PCR, quantitative PCR; shRNA, short hairpin RNA.

LNCaP cells cocultured with younger PZ stromal cells (Figure $3 \mathbf{b}$ and c). However, the difference of migration and invasion disappeared after AR expression was knocked down (Figure $3 \mathbf{b}$ and c). Regarding LNCaP cells cocultured with younger PZ stromal cells expressing AR-targeting shRNA, the rate of migration and invasion was more rapid than that of $\mathrm{LNCaP}$ cells cocultured with younger $\mathrm{PZ}$ stromal cells (Figure $\mathbf{3 b}$ and $\mathbf{c}$ ). By contrast, LNCaP cells cocultured with older PZ stromal cells expressing AR-targeting shRNA were observed to migrate and invade more slowly than LNCaP cells cocultured with older PZ stromal cells (Figure $3 \mathbf{b}$ and $\mathbf{c}$ ).

MMP expression varied following knockdown of the AR in prostate stromal cells

After AR expression was knocked down in stromal cells obtained from males of various ages, levels of MMP1, MMP2, MMP7 and MMP9 mRNA were detected by q-PCR. In these assays, expression levels of $M M P 1, M M P 2, M M P 7$ and MMP9 increased in the younger group samples (Figure 4). But in the older group the expression of MMP2 decreased and MMP9 increased (Figure $4 \mathbf{b}$ and d).

The prostatic stromal AR played different roles in the metastasis of PC3 cells in vivo depending on age

To study metastasis in vivo, a nude mouse model was used. Mice were separated into three experimental groups with nine mice per group. Stromal cells of varying ages with or without AR expression and PC3 cells $\left(5 \times 10^{6}\right)$ were mixed in a $1: 1$ ratio and suspended in $0.1 \mathrm{ml}$ of undiluted Matrigel (Becton Dickinson Biosciences). Cell mixtures were then injected into the left prostate anterior lobe of male athymic mice. After 8 weeks, one group of mice did not exhibit lymph node metastasis (Figure 5a), one group exhibited unilateral lymph node metastasis (Figure 5b) and a third group exhibited bilateral metastatic lymph nodes (Figure 5c). Despite these differences in metastasis 
A
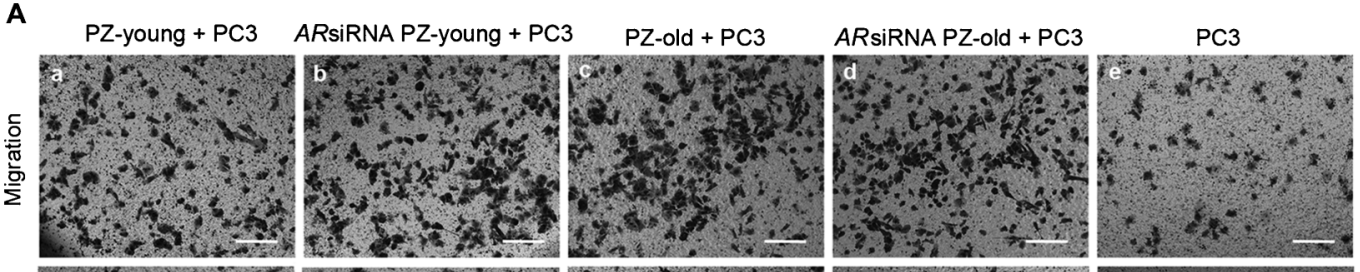
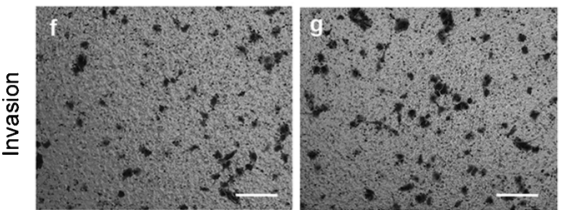

B

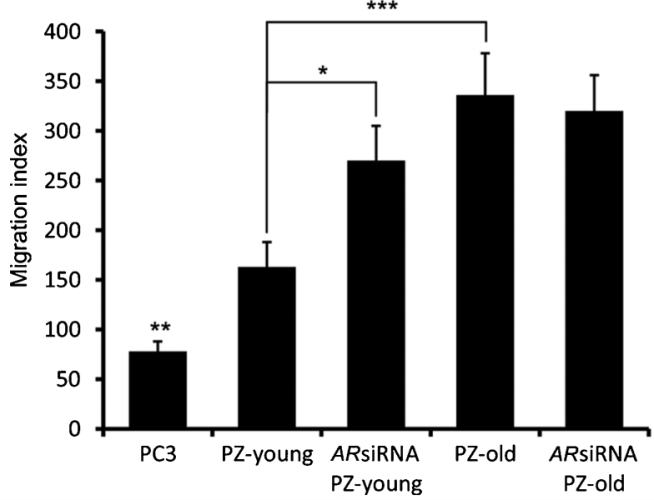

C

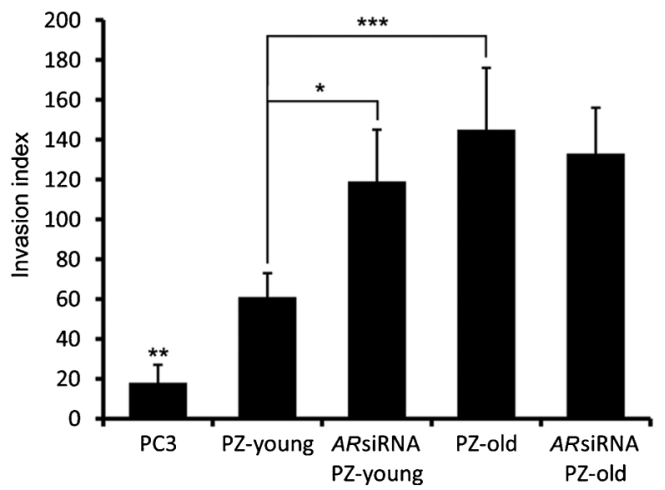

Figure 2 The effects of AR expression by prostatic stromal cells on the migration and invasiveness of PC3 cells. (A) The PC3 cells transferred through the membrane were observed through a microscope. (B,C) Knockdown of the AR in stromal cells obtained from both younger males and older males only promoted the migration and invasiveness of PC3 cells exposed to the former. Older PZ stromal cells promoted the migration and invasiveness of PC3 cells more than younger PZ stromal cells. However, these effects disappeared after AR expression was knocked down. All stromal cells were found to obviously promote the migration and invasiveness of PC3 cells. Each bar represents the number of transferred cells from three independent experiments. ${ }^{*} P<0.05$. (A) Scale bar $=20 \mu \mathrm{m}$. AR, androgen receptor; PZ, peripheral zone; siRNA, small interfering RNA.

development, the rate of tumour formation did not differ significantly among the five groups. After AR expression was knocked down in prostatic stromal cells, the incidence of metastatic lymph nodes was observed to increase in the younger age group (PZ-young + PC3 cells and PZ-young expressing AR shRNA + PC3 cells), but decreased in the older age group (PZ-old $+\mathrm{PC} 3$ cells and PZ-old expressing $A R$ shRNA+PC3 cells) (Table 1). In addition, the difference between the older age group (PZ-old+PC3 cells) and younger age group (PZ-young + PC3 cells) disappeared after knocking down the stromal AR (Table 1). Moreover, all stromal cells could promote PC3 metastasis in vivo (Table 1). Metastatic lymph nodes and transplanted tumours were verified by H\&E staining, and PC3 cells were observed all over the field of vision under the microscope (Figure 6a and c). Furthermore, immunohistochemistry assays detected a decrease in MMP2 expression following knockdown of the stromal AR in the older age group (Figure 6d), and an increase in MMP2 expression in the younger age group (Figure 6d). Levels of MMP2 detected in the PZ-old + PC3 cells group were also higher than in the PZ-young + PC3 cells group, although this difference in MMP2 expression was absent following knockdown of the AR in prostatic stromal cells (Figure 6d).

\section{DISCUSSION}

PCa represents a major health problem for men worldwide. However, the etiopathogenesis of PCa remains obscure. Interestingly, PCa was not reported for eunuchs who had their testicles resected in their youth during the Qing Dynasty. ${ }^{20}$ Moreover, $70 \%$ of PCa cases involve men over the age of 65 , whereas few cases involve males younger than 50 years. As a result, age has been identified as a risk factor for $\mathrm{PCa}{ }^{2}$ However, the age-associated decline in circulating levels of testosterone and intra-prostatic dihydrotestosterone levels seems paradoxical with respect to the higher occurrence of PCa reported in older men. ${ }^{21}$ In addition, low testosterone levels have been associated with worse clinical and pathological determinants of $\mathrm{PCa}$, including an increased risk of $\mathrm{PCa} .{ }^{22}$ Moreover, the $\mathrm{AR}$ has been found to play a critical role in prostate carcinogenesis.

In a recent article, it was demonstrated that the AR expressed by prostate stromal cells and not by epithelial cells, promoted PCa progression. ${ }^{12}$ In addition, the stromal cells from older males have been found to play a more aggressive role in PCa than stromal cells from younger males. ${ }^{5}$ In the present study, we found that the stromal AR could explain the different roles for stromal cells in different ages. For example, the AR in prostatic stromal cells obtained from younger males was associated with an inhibition of PC3/LNCaP metastasis. By contrast, the AR in prostatic stromal cells obtained from older males was associated with promotion of $\mathrm{PC} 3 / \mathrm{LNCaP}$ metastasis. These results provide a valuable viewpoint for understanding the carcinogenesis of PCa.

In the present study, we found that the expression of the AR in prostatic stromal cells did not differ between younger males and older males. Additionally, the particular issue was whether the role of the AR varied by aging. For example, Cunha et al. ${ }^{23}$ demonstrated that prostatic development and growth are mediated by signalling via stromal 


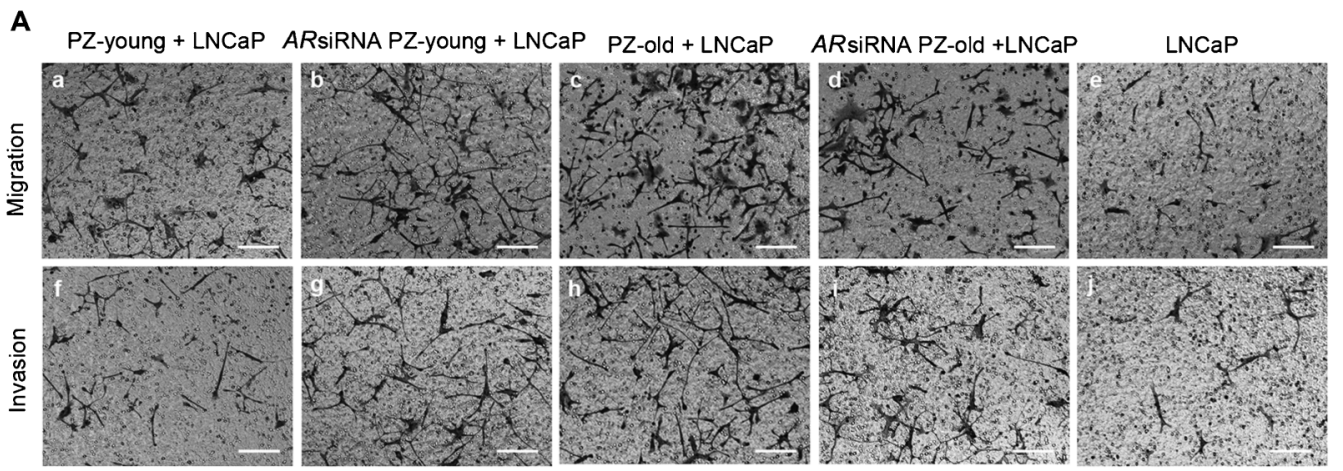

B

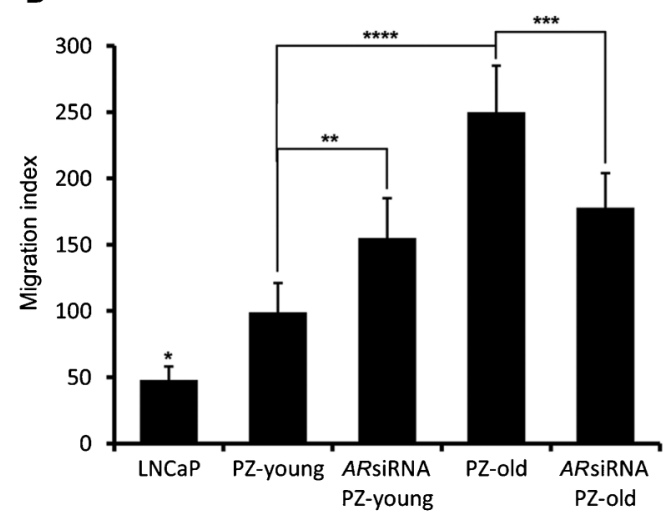

C

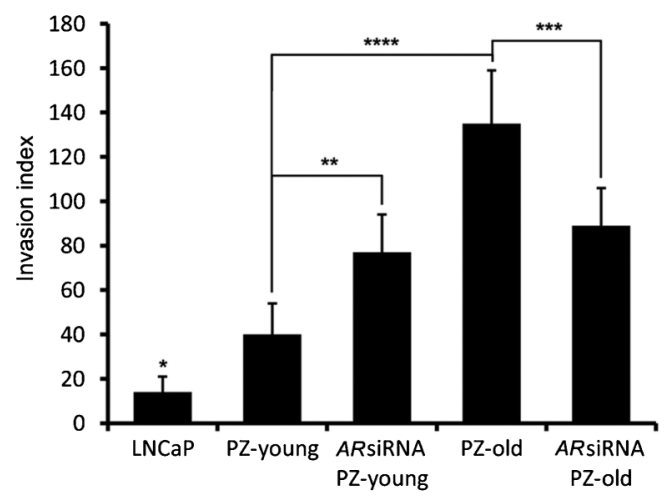

Figure 3 The effects of AR expression by prostatic stromal cells on the migration and invasiveness of LNCaP cells. (A) The LNCaP cells transferred through the membrane were observed through a microscope. (B,C) Knockdown of AR expression in stromal cells obtained from younger versus older males was found to promote versus inhibit the migration and invasiveness of $\mathrm{LNCaP}$ cells, respectively. Older PZ stromal cells promoted the migration and invasiveness of $\mathrm{LNCaP}$ cells more effectively than younger PZ stromal cells. However, these effects disappeared following the knockdown of AR expression (B,C). All stromal cells were found to obviously promote the migration and invasiveness of $L N C a P$ cells $(B, C)$. Each bar represents the number of transferred cells from three independent experiments. $* P<0.05$. (A) Scale bar $=20 \mu \mathrm{m}$. AR, androgen receptor; PZ, peripheral zone; siRNA, small interfering RNA.
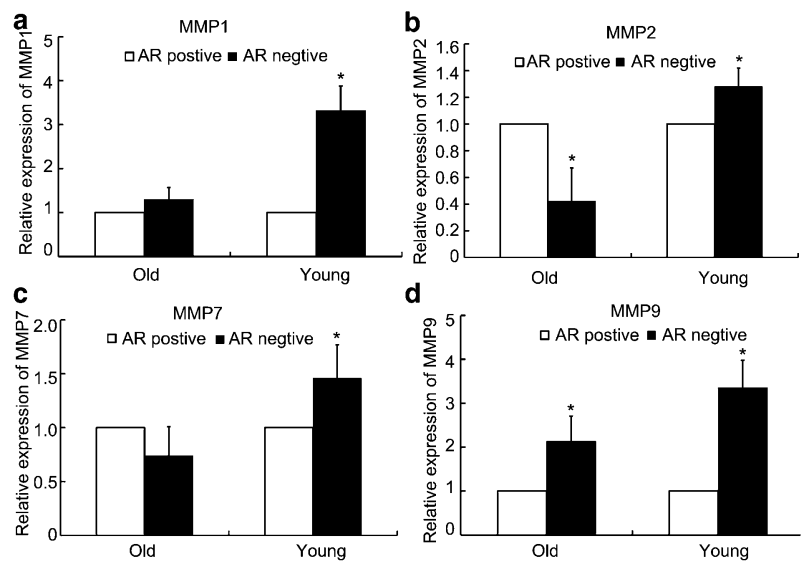

Figure 4 After AR expression was knocked down in prostatic stromal cells, changes in MMP expression were detected. (a) MMP1 was found to be upregulated in prostatic stromal cells obtained from younger males who were treated with AR-targeting shRNA. By contrast, MMP1 levels were unchanged in samples obtained from older males. (b, c) MMP2 and MMP7 were downregulated versus upregulated in prostatic stromal cells obtained from older versus younger males, respectively, who were treated with AR-targeting shRNA. (d) MMP9 was upregulated in prostatic stromal cells obtained from both older and younger males who were treated with AR-targeting shRNA, but the variance was larger in the younger group samples. $* P<0.05$. AR, androgen receptor; MMP, matrix metalloproteinase; shRNA, small hairpin RNA.
AR and not epithelial AR. In addition, Niu et al. ${ }^{24}$ reported that PCa progression is androgen dependent, and androgen dependency occurs via AR expression by prostatic stromal cells and not by AR expression by epithelial cells. In the present study, the PCa cells cocultured with stromal cells from older males were observed to be more aggressive than the PCa cells cocultured with stromal cells from younger males. The difference between the older and younger groups before knocking down the AR was also consistent with the results of our former study. ${ }^{6}$ Moreover, the AR expressed by prostatic stromal cells obtained from younger males was associated with inhibition of the migration of PC3/ LNCaP cells and invasiveness of LNCaP cells, and this was not observed in prostatic stromal cells obtained from older males (Figures $\mathbf{2}$ and 3). A potential explanation for these observations is that the function of the stromal AR changes according to age. Specifically, the role of the AR in stromal cells appears to change from inhibition to promotion of PCa metastasis.

Of the various stages involved in prostate carcinogenesis, cross-talk between stromal and epithelial cells represents a key component that is also complicated. The role of stromal cells in the metastasis of epithelial cells has been shown to primarily depend on exocrine factors such as MMPs that promote carcinogenesis. ${ }^{25}$ For example, Li et al. ${ }^{26}$ demonstrated that androgens can enhance MMP-2 promoter activity, and MMP2 is mainly expressed in the stromal cells of the prostate. ${ }^{27}$ In the present study, the AR in prostatic stromal cells contributed to the regulation of MMPs in prostatic stromal cells. Furthermore, stromal cells could produce MMPs that facilitate the migrating of tumour 

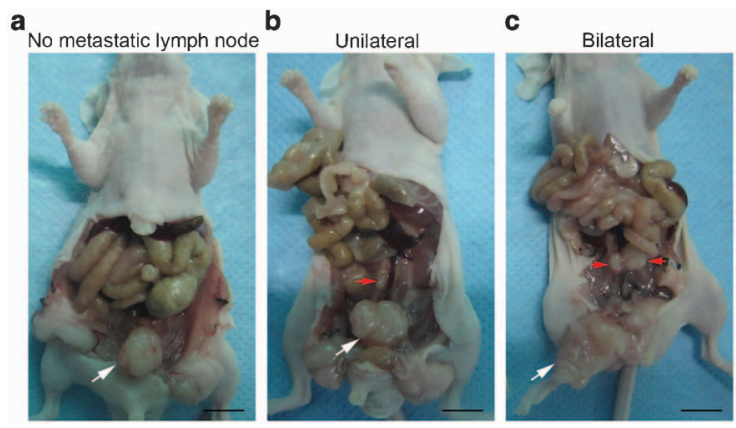

Figure 5 The effects of $\mathrm{AR}^{+/-}$stromal cells of varying ages on the metastasis of tumours in nude mice. For each experimental group, athymic mice were divided according to (a) the absence of metastatic lymph nodes or the presence of unilateral (b) or bilateral (c) metastatic lymph nodes. Lymph nodes are labelled with red arrows, whereas tumours are indicated with white arrows. AR, androgen receptor. cells. ${ }^{28}$ In the younger male group, knocking down the AR in prostatic stromal cells was accompanied by an increase in the expression of various MMPs. However, that observation was not noted in the older male group. These results suggest that the AR in prostatic stromal cells could inhibit the expression of MMPs in younger males but not in older males.

In general, MMPs have been shown to have well-recognized roles in the late stages of tumour progression, invasion, and metastasis. For example, MMP-2 and MMP-9 participate in the degradation of extracellular matrix components, including the basement membrane, which separates epithelia from the stroma. ${ }^{29}$ Moreover, constitutive expression of MMP7 has been shown to render cancer cells more invasive. ${ }^{30}$ Similarly, cleavage of osteopontin by MMP-9 generates a 5 - $\mathrm{kDa}$ fragment that promotes tumour cell invasion, ${ }^{21}$ whereas cleavage of E-cadherin by MMP-9 promotes epithelial mesenchymal transition. ${ }^{31}$ Furthermore, increased expression of MMP-1 and MMP-2 has been linked to lymphatic invasion and lymph node metastasis, ${ }^{32}$

Table 1 Role of kinds of stromal cells in tumour metastasis in a nude mouse model

\begin{tabular}{lccccc}
\hline Experimental group & Mice with formed tumour & Mice with unformed tumour & \multicolumn{3}{c}{ Metastatic retroperitoneal lymph nodes } \\
\cline { 3 - 6 } & & & No. & Unilateral & Bilateral \\
\hline PZ-young+PC3 & 13 & 1 & 3 & 9 & 1 \\
AR siRNA PZ-young+PC3 & 10 & 4 & 0 & 4 & 0 \\
PC3 & 11 & 3 & 9 & 2 & $0.0001^{*}$ \\
PZ-old+PC3 & 14 & 0 & 0 & 3 & 11 \\
AR siRNA PZ-old+PC3 & 12 & 2 & 2 & 7 & 3 \\
\hline
\end{tabular}

Abbreviations: AR, androgen receptor; PZ, peripheral zone; siRNA, small interfering RNA.

* Means each couple groups of the first three groups significantly differed.

** Means each couple groups of the last three groups significantly differed.

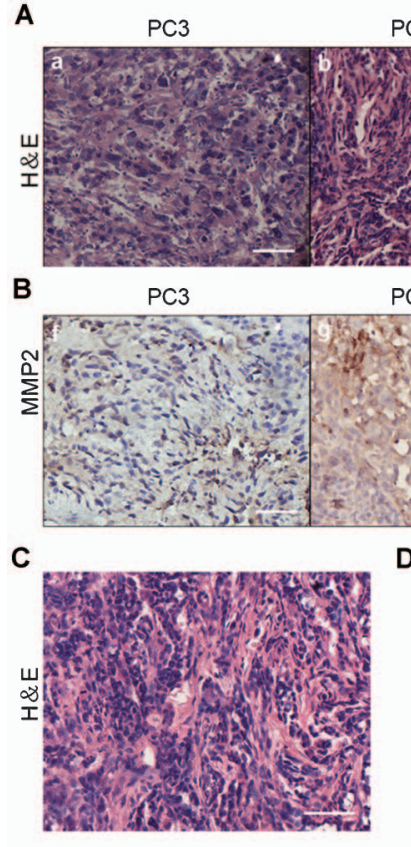

PC3+PZ-old

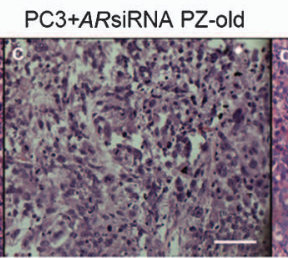

PC3+PZ-young PC3+ARsiRNA PZ-young

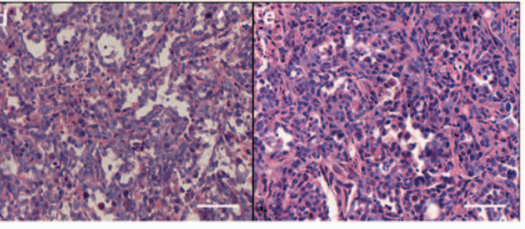

PC3+PZ-old

PC3+ARsiRNA PZ-old

$\mathrm{PC} 3+\mathrm{PZ}$-young

PC3+ARsiRNA PZ-young

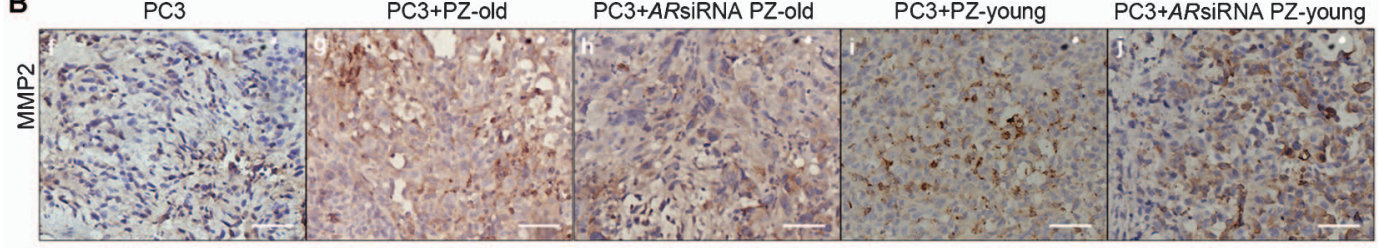

D

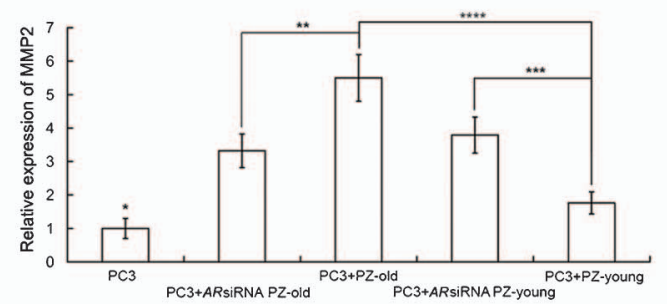

Figure 6 Transplanted tumours (A) and metastatic lymph nodes (C) were verified by H\&E staining. Expression of MMP2 was detected by immunohistochemistry for the five groups (B). Relative levels of MMP2 were compared between AR + and AR - groups for both the younger and older groups (D). Levels of MMP2 were observed to increase versus decrease following the knockdown of AR expression in prostatic stromal cells from the younger versus older group, respectively (D). The expression of MMP2 increased when PC3 cells were cocultured with various types of prostatic stromal cells (D). Each value represents the mean \pm s.d. of three independent experiments. $* P<0.05$. Scale bar $=20 \mu \mathrm{m}$. AR, androgen receptor; H\&E, haematoxylin and eosin; MMP, matrix metalloproteinase. 
whereas MMP-1 derived from tumour-infiltrating fibroblasts appears to drive cancer cell migration and invasive behaviour. MMPs have also been found to play key roles in the formation of a metastatic niche.

Work by Johansson et al. ${ }^{33}$ noted that the knockdown of AR expression by prostate stromal cells has the potential to inhibit carcinogenesis. Consequently, the knockdown of stromal AR expression may represent a potential therapeutic approach for the treatment of PCa. ${ }^{34}$ However, the cases associated with these results all involved males older than 50 years. In our opinion, these results do not directly translate for younger patients with PCa (e.g., <40 years), and further study is needed to characterize the different functions of the AR axis in stromal cells, particularly in relation to age.

In conclusion, the present study illustrates that expression of the AR by prostatic stromal cells of males of different ages can differentially affect PCa progression. Although prostatic stromal cells from both older and younger males could both promote PCa progression, the effectiveness of prostatic stromal cells obtained from older males was more evident. Moreover, AR expression by prostatic stromal cells from younger males appeared to inhibit PCa progression. A possible explanation for these observations is that the function of the AR axis in prostatic stromal cells changes according to age, and this finding represents a novel and valuable direction for future studies of $\mathrm{PCa}$ pathogenesis.

\section{AUTHOR CONTRIBUTIONS}

YYL and BJ designed and carried out the experiments, analysed the data and drafted the manuscript. BMH and SJX designed the experiments and wrote the paper. DC and QJ participated in performing the PCR assays, helped perform the statistical analyses and drafted the manuscript. FJZ, JJY, EHL and XHW participated in the collection of specimens and in primary cell culture. All authors read and approved the final manuscript.

\section{COMPETING FINANCIAL INTERESTS}

The authors declare no competing financial interests.

\section{ACKNOWLEDGMENTS}

This work was supported by the Innovation Program of the Shanghai Municipal Education Commission (No. 102216) and by the National Natural Science Foundation of China (No. 81072096 and No. 81072114).

1 Jemal A, Bray F, Center MM, Ferlay J, Ward E et al. Global cancer statistics. CA Cancer J Clin 2011; 61: 69-90.

2 Ahmedin Jemal RS, Xu JQ, Ward E. Cancer statistics, 2010. CA Cancer J Clin 2010; 60: 277-300.

3 Barclay WW, Woodruff RD, Hall MC, Cramer SD. A system for studying epithelialstromal interactions reveals distinct inductive abilities of stromal cells from benign prostatic hyperplasia and prostate cancer. Endocrinology 2005; 146: 13-8.

4 Cunha GR, Hayward SW, Wang YZ, Ricke WA. Role of the stromal microenvironment in carcinogenesis of the prostate. Int J Cancer 2003; 107: 1-10.

5 Wang YC, Yu SQ, Wang XH, Han BM, Zhao FJ et al. Differences in phenotype and gene expression of prostate stromal cells from patients of varying ages and their influence on tumour formation by prostate epithelial cells. Asian J Androl 2011; 13: 732-41.

6 Mohler JL. A role for the androgen-receptor in clinically localized and advanced prostate cancer. Best Pract Res Clin Endocrinol Metab 2008; 22: 357-72.

7 Cohen MB, Rokhlin OW. Mechanisms of prostate cancer cell survival after inhibition of AR expression. J Cell Biochem 2009; 106: 363-71.
8 Edwards J, Krishna NS, Grigor KM, Bartlett JM. Androgen receptor gene amplification and protein expression in hormone refractory prostate cancer. Br J Cancer 2003; 89 : 552-6.

9 Cheng H, Snoek R, Ghaidi F, Cox ME, Rennie PS. Short hairpin RNA knockdown of the androgen receptor attenuates ligand-independent activation and delays tumor progression. Cancer Res 2006; 66: 10613-20.

10 Liao X, Tang S, Thrasher JB, Griebling TL, Li B. Small-interfering RNA-induced androgen receptor silencing leads to apoptotic cell death in prostate cancer. $\mathrm{Mol}$ Cancer Ther 2005; 4: 505-15.

11 Berry PA, Maitland NJ, Collins AT. Androgen receptor signalling in prostate: effects of stromal factors on normal and cancer stem cells. Mol Cell Endocrinol 2008; 288: 30-7.

12 Ricke EA, Williams K, Lee YF, Couto S, Wang Y et al. Androgen hormone action in prostatic carcinogenesis: stromal androgen receptors mediate prostate cancer progression, malignant transformation and metastasis. Carcinogenesis 2012; 33: 1391-8.

13 Henshall SM, Quinn DI, Lee CS, Head DR, Golovsky D et al. Altered expression of androgen receptor in the malignant epithelium and adjacent stroma is associated with early relapse in prostate cancer. Cancer Res 2001; 61: 423-7.

14 Ricciardelli C, Choong CS, Buchanan G, Vivekanandan S, Neufing P et al. Androgen receptor levels in prostate cancer epithelial and peritumoral stromal cells identify nonorgan confined disease. Prostate 2005; 63: 19-28.

15 Halin S, Hammarsten P, Wikstrom P, Bergh A. Androgen-insensitive prostate cancer cells transiently respond to castration treatment when growing in an androgendependent prostate environment. Prostate 2007; 67: 370-7.

16 Karlou M, Tzelepi V, Efstathiou E. Therapeutic targeting of the prostate cancer microenvironment. Nat Rev Urol 2010; 7: 494-509.

17 Zhao FJ, Han BM, Yu SQ, Xia SJ. Tumor formation of prostate cancer cells influenced by stromal cells from the transitional or peripheral zones of the normal prostate. Asian J Androl 2009; 11: 176-82.

18 Livak KJ, Schmittgen TD. Analysis of relative gene expression data using real-time quantitative PCR and the 2(-Delta Delta $C_{\mathrm{T}}$ ) Method. Methods 2001; 25: 402-8.

19 Chadha KC, Nair BB, Chakravarthi S, Zhou R, Godoy A, Mohler JL et al. Enzymatic activity of free-prostate-specific antigen ( $f-P S A$ ) is not required for some of its physiological activities. Prostate 2011 ; 71: 1680-90.

$20 \mathrm{Wu}$ JP, Gu FL. The prostate $41-65$ years post castration. An analysis of 26 eunuchs. Chin Med J (Engl) 1987; 100: 271-2.

21 Sampson N, Untergasser G, Plas E, Berger P. The ageing male reproductive tract. J Pathol 2007; 211: 206-18.

22 Pierorazio PM, Ferrucci L, Kettermann A, Longo DL, Metter EJ et al. Serum testosterone is associated with aggressive prostate cancer in older men: results from the Baltimore Longitudinal Study of Aging. BJU Int 2010; 105: 824-9.

23 Cunha GR, Lung B. The possible influence of temporal factors in androgenic responsiveness of urogenital tissue recombinants from wild-type and androgeninsensitive (Tfm) mice. J Exp Zool 1978; 205: 181-93.

24 Niu Y, Altuwaijri S, Yeh S, Lai KP, Yu S et al. Targeting the stromal androgen receptor in primary prostate tumors at earlier stages. Proc Natl Acad Sci USA 2008; 105 : 12188-93.

25 Egeblad M, Werb Z. New functions for the matrix metalloproteinases in cancer progression. Nat Rev Cancer 2002; 2: 161-74.

26 Li BY, Liao XB, Fujito A, Thrasher JB, Shen FY et al. Dual androgen-response elements mediate androgen regulation of MMP-2 expression in prostate cancer cells. Asian $\mathrm{J}$ Androl 2007; 9: 41-50.

27 Zhang J, Jung K, Lein M, Kristiansen G, Rudolph B et al. Differential expression of matrix metalloproteinases and their tissue inhibitors in human primary cultured prostatic cells and malignant prostate cell lines. Prostate 2002; 50: 38-45.

28 Donadio AC, Durand S, Remedi MM, Frede S, Ceschin DG et al. Evaluation of stromal metalloproteinases and vascular endothelial growth factors in a spontaneous metastasis model. Exp Mol Pathol 2005; 79: 259-64.

29 Feng G, Tan Y. Expression and significance of MMP2 and type IV collagen in gastric cancer. Zhonghua Wai Ke Za Zhi 2000; 38: 775-7, 745.

30 Fingleton B, Vargo-Gogola T, Crawford HC, Matrisian LM. Matrilysin [MMP-7] expression selects for cells with reduced sensitivity to apoptosis. Neoplasia 2001; 3: 459-68.

31 Tester AM, Cox JH, Connor AR, Starr AE, Dean RA et al. LPS responsiveness and neutrophil chemotaxis in vivo require PMN MMP-8 activity. PLOS ONE 2007; 2 . e312.

32 Langenskiold M, Holmdahl L, Falk P, Ivarsson ML. Increased plasma MMP-2 protein expression in lymph node-positive patients with colorectal cancer. Int J Colorectal Dis $2005 ; 20: 245-52$

33 Johansson A, Jones J, Pietras K, Kilter S, Skytt A et al. A stroma targeted therapy enhances castration effects in a transplantable rat prostate cancer model. Prostate 2007; 67: 1664-76.

34 Yu S, Yeh CR, Niu Y, Chang HC, Tsai YC et al. Altered prostate epithelial development in mice lacking the androgen receptor in stromal fibroblasts. Prostate 2012; 72: 43749. 